\title{
Data Intensive Imaging for 3D Atom Probe
}

\author{
Scott R. Broderick, Santoshrupa Dumpala, Sonal Padalkar, Krishna Rajan \\ Department of Materials Science and Engineering and Institute for Combinatorial Discovery, Iowa State \\ University, 2220 Hoover Hall, Iowa State University, Ames, IA 50011
}

Atom Probe Tomography (APT) combines atomic imaging with a time-of-flight mass spectrometer to provide direct space three-dimensional, atomic-scale resolution images of materials with the chemical identities of all the detected atoms. Quantitative analysis of APT data through advanced data mining methods enhances the chemical characterization of alloy systems and accounts for the statistical uncertainty associated with missing data and the physics of preferential evaporation. Most techniques currently used to extract precipitate topology and interface information from APT data are efficient; however, they are based on homogenization of the rich point cloud data which inherently loses some information. Furthermore, these methods require a specified, usually heuristic, concentration-level to draw iso-contours in order to extract characteristics of the precipitate topology. These twin issues of homogenization and heuristics are compelling rationale for the development of a robust, scalable, heuristic-free, graph-based framework [1].

Through data mining, we improve the resolution of the mass spectra, quantify chemical surfaces in terms of their topological features, and integrate visualization and modeling approaches to reduce effects of noise in APT [2]. In particular, we address visualization challenges associated with the volume (hundreds of millions of gigabytes and ions collected) and the velocity (five million ions per minute) of the data collected. We introduce an approach for visualizing all of the atoms measured in the material, while current standards are only a few percent of the ions, by splitting rendering tasks between the central processing unit and the graphics processing unit (Figure 1). Through this enhanced visualization, we quantify uncertainty in the data as a function of spatial distribution. This data representation captures the physics of APT while addressing the imaging challenges associated with the data volume and velocity. This work addresses how the techniques associated with large scale data analytics coupled to experimental data can provide quantitative insight into the interpretation of atomic scale chemical imaging

The present work is aimed at developing quantitative techniques for the detection of sharp chemical interfaces from the APT outputs of 3D point cloud and 3D chemical interfaces of continuous geometry. The difficulty lies in mapping discrete data to a continuous output while minimizing the loss in chemical information. In the present work, we develop a novel approach, based on the principles of computational homology, to map the discrete 3D point cloud atomic data to the topology of a continuous chemical interface. The computational homology framework developed can be applied to APT data to get meaningful results and quantify sensitivity of the output on the data reconstruction parameters. To incorporate evaporation physics with chemistry and structure and to provide physical definition of uncertainty in spatially defining phases, ion evaporation maps are employed (Figure 2) [3]. The differences in evaporation physics at the bulk and interface provide an alternative approach to defining the chemical interface [4].

[1] S Samudrala et al, Computational Materials Science 77 (2013), p. 335.

[2] S Broderick et al, Ultramicroscopy 132 (2013), p. 121.

[3] J Peralta, S Broderick, K Rajan, Ultramicroscopy 132 (2013), p. 143. 
[4] Support comes from Air Force Office of Scientific Research grants: FA9550-11-1-0158 and FA9550-12-0496; and NSF ARI Program grant: CMMI-09-389018.

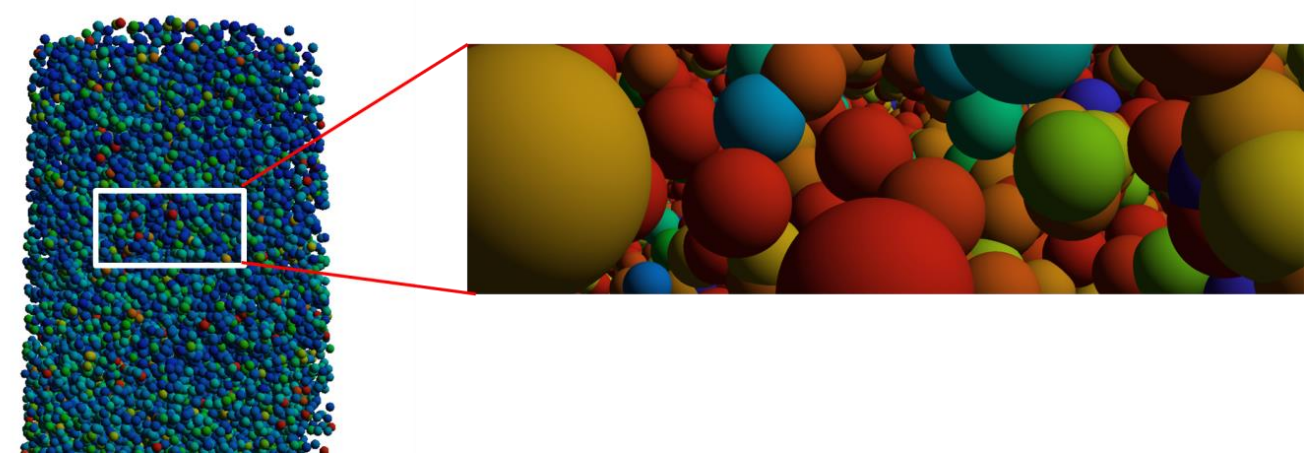

Figure 1. 3D visualization of APT data, where $100 \%$ of collected atoms are rendered. By visualizing all of the data and addressing spatial uncertainty, we assess the impact of all collected attributes on the data fidelity and uncertainty.
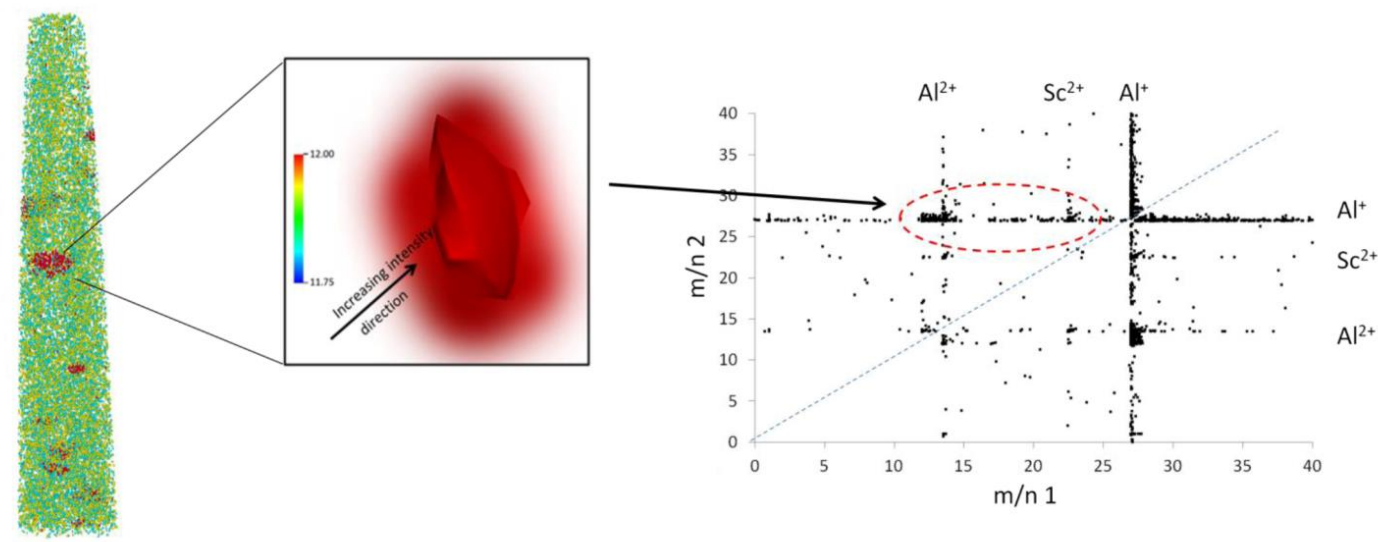

Figure 2. Uncertainty in heuristic-free defined precipitate topology is defined based on linking 3D point cloud data with continuously mapped chemical data. These outputs are linked with ion evaporation map, linking evaporation physics with chemistry and frequency, while addressing the visualization challenges associated with APT data volume and velocity. The statistical analysis of this data provides new insights into chemical bonding. 